\title{
optica
}

\section{Total absorption of visible light in ultrathin weakly absorbing semiconductor gratings: supplementary material}

\author{
Björn C. P. Sturmberg 1,6, , Teck K. Chong 2,6 , Duk-Yong Chol ${ }^{2}$, Thomas P. \\ White ${ }^{2}$, Lindsay C. Botten ${ }^{3,4}$, Kokou B. Dossou ${ }^{4}$, Christopher G. \\ Poulton $^{4}$, Kylie R. Catchpole ${ }^{2}$, Ross C. McPhedran ${ }^{1}$, and C. Martijn de \\ STERKE ${ }^{1}$
}

${ }^{1}$ CUDOS and IPOS, School of Physics, University of Sydney, Sydney, 2006, Australia

${ }^{2}$ Centre for Sustainable Energy Systems, Research School of Engineering, Australian National University, Canberra, 2601, Australia

${ }^{3}$ National Computational Infrastructure, Australian National University, Canberra, 2601, Australia

${ }^{4}$ CUDOS, School of Mathematical and Physical Sciences, University of Technology Sydney, Sydney, 2007, Australia

${ }^{5}$ Laser Physics Centre, Research School of Physics and Engineering, Australian National University, Canberra, 2601, Australia

${ }^{6}$ Equal contribution first authors

*Corresponding author: bjorn.sturmberg@sydney.edu.au

Published 24 May 2016

\begin{abstract}
This document provides supplementary information to "Total absorption of visible light in ultrathin weakly absorbing semiconductor gratings," http://dx.doi.org/10.1364/optica.3.000556. Additional information regarding sample fabrication, optical characterization, and the effects of non-ideal metal back reflectors. Critical coupling derivation of Eq. 1, derivation of Eq. 5 and proof of the invariance of the properties of ultrathin gratings for constant $\varepsilon h / \lambda$. (C) 2016 Optical Society of America
\end{abstract}

\section{ABSORPTION BANDWIDTH}

Figures $\mathrm{S} 1(\mathrm{a})$ and $\mathrm{S} 1(\mathrm{~b})$ show the dependence of the absorption bandwidths ( $A=90 \%, A=95 \%$ respectively) on the loss tangent $(\operatorname{Im}(\varepsilon) / \operatorname{Re}(\varepsilon))$ of the gratings. The gratings are arranged in the asymmetric configuration with an optimised spacer layer and reflector, and the rulings have $|\epsilon|=14$. The target wavelength is $\lambda_{0}=600$ and $h=20,25,30$ for the blue lines with circle markers, the green lines with star markers, and for the red lines with triangle markers respectively. For $\operatorname{Im}(\varepsilon) / \operatorname{Re}(\varepsilon)<2$ the dependence is shown to be approximately linear, while at larger values of $\operatorname{Im}(\varepsilon) / \operatorname{Re}(\varepsilon)$ the bandwidth saturates. The absorptance spectra of gratings with $|\varepsilon| h / \lambda_{0}=0.7$ are shown in Fig. S2(a). The absorption spectrum of a homogenius absorbing layer optimised for the same wavelength $(\epsilon=0+3 i, h / \lambda=30 / 600$ in the asymmetric configuration) is shown in Fig. S2(b). The $|\epsilon|$ of the homogenius layer is significantly smaller than that of the gratings because the effective permittivity of the gratings is reduced by the air inclusions.

\section{EFFECT OF AN IMPERFECT METAL REFLECTOR}

Figures S3(a) and S4(a) show the absorption of a $h=\lambda / 70$ thick uniform layer illuminated from one side, with a spacer layer and reflector on the other side. This absorber layer is therefore the same as shown in Fig. 3(a) of the main text, except that it in arranged in the asymmetric configuration of Fig. 1(e) rather Fig. 1(c). In Fig. S3(a) the reflector is made of a perfect conductor and in and S4(a) it is made of silver. TLA occurs at a smaller $|n|$ than in the case of coherent illumination from both sides (Fig. 3(a)) because the light entering the layer from below has a lower amplitude than the light incident from above (it has passed through the absorber at least once). The condition $n^{\prime} \sim n^{\prime \prime}$ is preserved because it is a fundamental requirement of critically coupling the standing wave inside the absorber with the incident propagating wave. 

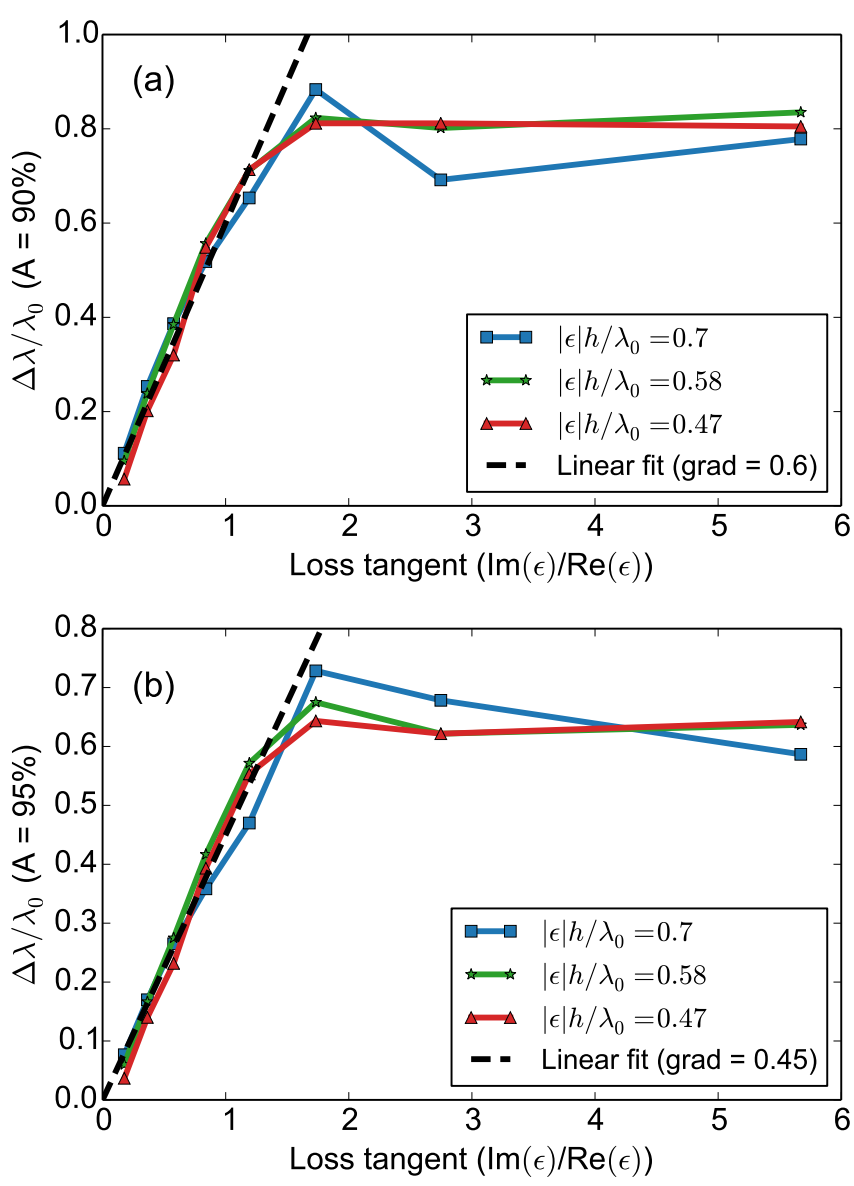

Fig. S1. $A=90 \%, A=95 \%$ absorption bandwidths, (a) and (b) respectively, of gratings as a function of their loss tangent. Line types indicate results for different values of $|\varepsilon| h / \lambda$.
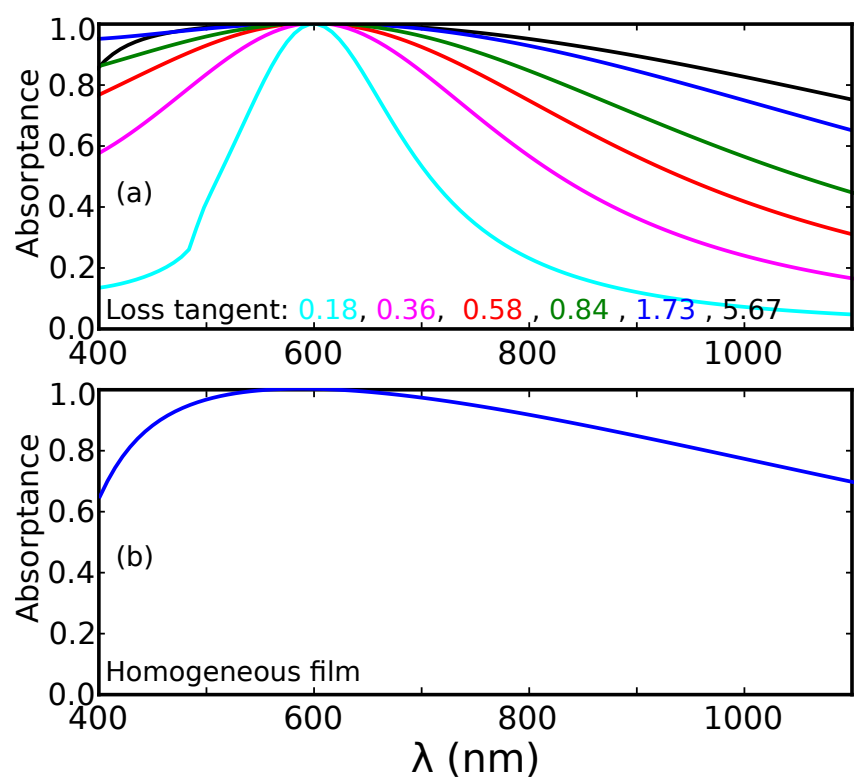

Fig. S2. (a) Absorptance spectra of gratings with $|\varepsilon| h / \lambda=0.7$ corresponding to the blue line with circle markers in Fig. S1(a) and S1(b). (b) Absorptance spectrum of a homogenius absorbing layer optimised for the same $\lambda_{0}=600 \mathrm{~nm}$.


Fig. S3. (a) Absorption as a function of complex refractive index for a uniform film with $h=\lambda / 70$, placed above a perfect conductor with a spacer layer of refractive index $m_{\mathrm{s}}$ and a thickness $h_{\mathrm{s}}$ shown in (b). The thickness of the spacer layer has been optimized to maximize the absorption. The TLA point lies slightly below the diagonal dashed line, where $h_{\mathrm{s}}=$ $\lambda / 4 m_{\mathrm{s}}$.


Fig. S4. (a) Absorption as a function of complex refractive index for a uniform film with $h=\lambda / 70$, placed above a silver reflector with a spacer layer of refractive index $m_{\mathrm{S}}$ and a thickness $h_{\mathrm{s}}$ shown in (b). The thickness of the spacer layer has been optimized to maximize the absorption. The TLA point lies slightly below the diagonal dashed line, where $h_{\mathrm{s}}<\lambda / 4 m_{\mathrm{s}}$.

For each value of $n$ we numerically optimize the thickness of the spacer layer, $h_{\mathrm{s}}$, which are presented in Figs. S3(b) and S4(b) for the perfect conductor and silver back reflectors respectively. In the case of the perfect conductor the optimal spacer layer thickness is $h_{\mathrm{s}}=\lambda / 4 m_{\mathrm{s}}$ (indicated by the red curve). Silver however is not an ideal metal at $\lambda \sim 600 \mathrm{~nm}$, and while its reflection coefficient has a magnitude close to unity, its phase is not precisely $\pi$, as it is for a perfect conductor. The spacer thickness required for TLA (which occurs at a value of $n$ just below the diagonal) is $h_{\mathrm{s}}<\lambda / 4 m_{\mathrm{s}}$.

\section{SAMPLE FABRICATION}

The $\mathrm{Sb}_{2} \mathrm{~S}_{3}$ grating stack was fabricated in the following way. Ag of $h=130 \mathrm{~nm}$ was thermally deposited on top of a polished silicon substrate at a deposition rate of $3 \AA . \mathrm{s}^{-1}$ using an Angstrom thermal evaporator. Subsequently, a $245 \mathrm{~nm}$ thick $\mathrm{SiO}_{2}$ film was deposited using an Oxford Plasmalab 100 dual frequency plasma-enhanced-chemical-vapour-deposition (PECVD) tool at a temperature of $300^{\circ} \mathrm{C}$ and deposition rate of $0.92 \mathrm{~nm} . \mathrm{s}^{-1}$. The resulting $\mathrm{SiO}_{2}$ film has a refractive index $n$ of 1.47 at $\lambda=600 \mathrm{~nm}$, measured by ellipsometry. A thin layer of $\mathrm{Sb}_{2} \mathrm{~S}_{3}$ was then thermally evaporated on top of the $\mathrm{SiO}_{2}$ film using an Angstrom system with a deposition rate of $0.3 \AA$ A.s ${ }^{-1}$. The resulting $\mathrm{Sb}_{2} \mathrm{~S}_{3}$ film thickness was $41 \mathrm{~nm}$.

To form the grating, a layer of positive photo resist (950 
PMMA A4) was spin-coated onto the stack $\left(\mathrm{Si} / \mathrm{Ag} / \mathrm{SiO}_{2} / \mathrm{Sb}_{2} \mathrm{~S}_{3}\right)$ and baked on a hotplate for 3 minutes at $180^{\circ} \mathrm{C}$. Gratings of size $100 \mu \mathrm{m} \times 100 \mu \mathrm{m}$ with varying period and line width were exposed in the PMMA using a Raith 150 electron-beam-lithography (EBL) system with a writing dose of $200 \mu \mathrm{C} . \mathrm{cm}^{-2}$. The exposed patterns were developed in MIBK/IPA developer solution for 1 minute followed by an IPA rinse to prevent over-development.

$\mathrm{Sb}_{2} \mathrm{~S}_{3}$ can be etched using inductively-coupled-plasma (ICP) etch using $\mathrm{CHF}_{3}$ gas. A range of etching parameters was initially trailed on test patterns to identify the conditions for etching a thin layer of $\mathrm{Sb}_{2} \mathrm{~S}_{3}$. The optimal etching recipe was found to be: pressure $=15 \mathrm{mTorr}$, ICP power $=200 \mathrm{~W}$, RF power $=75 \mathrm{~W}$ and $\mathrm{CHF}_{3}$ flow rates of $50 \mathrm{sccm}$. With these conditions $60 \mathrm{~s}$ is sufficient to remove $41 \mathrm{~nm}$ of $\mathrm{Sb}_{2} \mathrm{~S}_{3}$. Following the etching, the remaining PMMA was removed using oxygen plasma etch for 15 minutes.

\section{OPTICAL CHARACTERIZATION}

The grating reflectance spectra were measured in two steps. First, the reflectance of a large $\left(\sim 1 \mathrm{~cm}^{2}\right)$ unpatterned area of the sample was measured in a spectrophotometer with an integrating sphere attachment (Perkins Elmer). This provided an accurate $( \pm 1.3 \%)$ reflectance reference for calibrating the small-area reflectance measurements on the patterned areas of the sample.

The patterned sample was measured in a WITec 300s confocal microscope using a $20 \times, \mathrm{NA}=0.4$ objective lens for illumination and collection of reflected light. Illumination was provided by a broadband supercontinuum source (Fianium SC450-2) that was filtered using an acousto-optical tunable filter (AOTF) fibercoupled to the microscope. The AOTF was used to select eight closely-spaced wavelengths at a time for reflectance measurements, and measurements were repeated with different illumination wavelengths in order to span the spectral range of interest from $\lambda=520 \mathrm{~nm}$ to $\lambda=640 \mathrm{~nm}$. Reflected light was collected by a $100 \mu \mathrm{m}$ multimode fiber and coupled into a NIR spectrometer (Princeton Instruments) for detection. Spectra of patterned and unpatterned regions were measured in this way and calibrated using the large-area reference reflectance data to obtain the grating reflectance. Reflectance measurements were repeated on several occasions using multiple samples and found to be highly reproducible. The main source of uncertainty in the reflectance measurements in the vicinity of the absorption peak is thought to be random fluctuations in the source intensity and/or spectrum. Based on analysis of the raw spectral data, we estimate the accuracy of the measured reflectance values to be $\pm 0.5 \%$.

In Fig. S6 we present the reflection spectra of four $\mathrm{Sb}_{2} \mathrm{~S}_{3}$ grating structures, all of thickness $h_{\mathrm{s}}=41 \mathrm{~nm}$ (measured shown as symbols and simulated shown as curves). The gratings with reflection minima at $\lambda=591 \mathrm{~nm}, \lambda=605 \mathrm{~nm}$ are the same as in the main text, while those with reflection minima at $\lambda=595 \mathrm{~nm}$, $\lambda=610 \mathrm{~nm}$ have $d=380 \mathrm{~nm}, f \sim 73 \%$, and $d=390 \mathrm{~nm}$, $f \sim 75 \%$ respectively.

\section{CRITICAL COUPLING}

In this section we show that critical coupling is consistent with Eq. 1 in the main text. Consider a uniform film of refractive index $n=n^{\prime}+\mathrm{i} n^{\prime \prime}$ and thickness $h / 2$, enclosed on one side by a perfect mirror, and bounded on the other by a medium of refractive index $m$.

Photons oscillating within the layer strike the interface between media $n, m$ every $h n^{\prime} / c$ seconds, where $c$ is the speed of

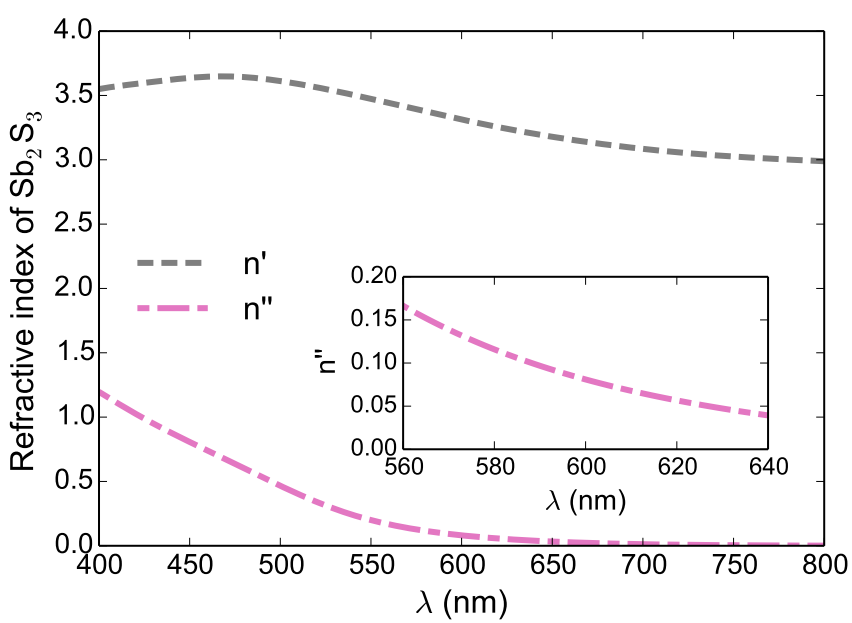

Fig. S5. The real $\left(n^{\prime}\right)$ and imaginary $\left(n^{\prime \prime}\right)$ parts of the refractive index of $\mathrm{Sb}_{2} \mathrm{~S}_{3}$, as measured using ellipsometry. A thin layer ( $h \sim 60 \mathrm{~nm}$ ) of $\mathrm{Sb}_{2} \mathrm{~S}_{3}$ was deposited directly on top of a polished silicon wafer. The band gap energy of the evaporated $\mathrm{Sb}_{2} \mathrm{~S}_{3}$ is $\sim 1.82 \mathrm{eV}$, corresponding to $\lambda=680 \mathrm{~nm}$. For a $41 \mathrm{~nm}$ thick $\mathrm{Sb}_{2} \mathrm{~S}_{3}$ film the single pass absorption at $\lambda=605 \mathrm{~nm}$ is $6.1 \%$ and $11.9 \%$ for double pass absorption at the vicinity of reflectance minimum.



Fig. S6. Reflectance of various gratings and the reference planar structure. Measured results are shown as symbols and simulated results are shown as curves, which are referred to in the legend by the wavelength of their reflectance minimum. In the interest of clarity we only present measurements close to the reflectance minima at $\lambda=595 \mathrm{~nm}$ and $\lambda=610 \mathrm{~nm}$. 
light in vacuum. The probability of a photon remaining within the layer after striking the interface is $\left|r^{\prime}\right|^{2}$, with $r^{\prime}$ defined in the main text. The probability of the same photon being within the layer after another round trip (at a time $t=\left(h n^{\prime} / \mathrm{c}\right)$ seconds later) is $\left|r^{\prime}\right|^{4}$. The probability of the photon remaining within the layer therefore decays exponentially as $\rho_{1}(t)=\exp \left(-t / \tau_{1}\right)$, where the decay constant $\tau_{1}$ is found from,

$$
\begin{aligned}
\rho_{1}(t)=\left(\left|r^{\prime}\right|^{2}\right)^{\mathrm{ct} / n^{\prime} h} & =e^{\ln \left(\left|r^{\prime}\right|^{2}\right) \mathrm{c} t / n^{\prime} h} \\
& =e^{\ln \left(1-\left(1-\left|r^{\prime}\right|^{2}\right)\right) c t / n^{\prime} h} \\
& =e^{-\ln \left(1-\left|r^{\prime}\right|^{2}\right) \mathrm{c} t / n^{\prime} h} .
\end{aligned}
$$

Therefore $\tau_{1}=-n^{\prime} h / \operatorname{cln}\left(\left|r^{\prime}\right|^{2}\right)$.

Meanwhile the probability of a photon remaining within the film also decays exponentially due to absorption. Disregarding losses due to photons escaping the thin film cavity, the probability of a photon being remaining in the layer is

$$
\begin{aligned}
\rho_{2}(t) & =e^{-4 \pi n^{\prime \prime} h / \lambda} \\
& =e^{-4 \pi n^{\prime \prime} \mathrm{c} t / \lambda n^{\prime}},
\end{aligned}
$$

so that the associated decay constant is $\tau_{2}=n^{\prime} \lambda / 4 \pi n^{\prime \prime} \mathrm{c}$.

At critical coupling $\tau_{1}=\tau_{2}$, so setting $e^{\tau_{1}}=e^{\tau_{2}}$ we find,

$$
\begin{aligned}
e^{n^{\prime} \lambda / 4 \pi n^{\prime \prime} \mathrm{c}} & =e^{-n^{\prime} h / \ln \left(\left|r^{\prime}\right|^{2}\right)} \\
e^{-4 \pi n^{\prime \prime} h / \lambda} & =e^{\ln \left(\left|r^{\prime}\right|^{2}\right)} \\
|\gamma|^{4} & =|r|^{2},
\end{aligned}
$$

using the definition of $\gamma$ and the relationship of the Fresnel coefficients that $\left|r^{\prime}\right|=|r|$. Equation 8 is consistent with Eq. 1 from the main text, although it does not contain information about the relative signs of $r$ and $\gamma^{2}$.

\section{DERIVATION OF EQ. 5}

The Taylor expansion of the tangent function for small arguments up to third order is $\tan (x)=x+x^{3} / 3$. The first order expansion of Eq. 4 in the main text is therefore

$$
n=\frac{\mathrm{i} m}{n k_{0} h / 2}
$$

while to third order we have

$$
n=\frac{\mathrm{i} m}{n k_{0} h / 2+\frac{1}{3}\left(n k_{0} h / 2\right)^{3}} .
$$

Upon rearranging this is

$$
\mathrm{i} m=n^{2} k_{0} h / 2+\frac{1}{3}\left(k_{0} h / 2\right)^{3} n^{4},
$$

and substituting in Eq. 9 for $n^{4}$,

$$
\begin{aligned}
\mathrm{i} m & =n^{2} k_{0} h / 2+\frac{1}{3}\left(k_{0} h / 2\right)^{3}\left(\frac{-4 m^{2}}{k_{0}^{2} h^{2}}\right), \\
& =n^{2} k_{0} h / 2-\frac{1}{3}\left(k_{0} h / 2\right) m^{2}, \\
n^{2} & =\frac{2 \mathrm{i} m}{k_{0} h}+\frac{1}{3} m^{2}, \\
& =\frac{\mathrm{i} m \lambda}{h \pi}+\frac{1}{3} m^{2},
\end{aligned}
$$

which is Eq. 5 in the main text.

\section{DERIVATION OF GRATING SCALING LAW}

We consider TE or $E_{\|}$polarization in which the electric field is aligned with the grooves of a lamellar grating of thickness $h$, located between $-h / 2 \leq z \leq h / 2$. Above and below the grating, the field is written in the respective plane wave expansions

$$
\begin{aligned}
& E(x, y)=\sum_{p} \eta_{p}^{-1 / 2}\left(\delta_{p} \exp \left(-i \chi_{p} z\right)+r_{p} \exp \left(i \chi_{p} z\right)\right) e_{p}(x) \\
& E(x, y)=\sum_{p} \eta_{p}^{-1 / 2} t_{p} \exp \left(-i \chi_{p} z\right) e_{p}(x)
\end{aligned}
$$

where $\delta_{p}, r_{p}$ and $t_{p}$ denote the incident, reflected and transmitted field amplitudes, $e_{p}=\exp \left(i \alpha_{p} x\right) / \sqrt{d}$ denotes the normalised plane wave Bloch field, $\alpha_{p}=\alpha_{0}+2 \pi p / d, \chi_{p}=\sqrt{k^{2}-\alpha_{p}^{2}}$, and $\eta_{p}=\chi_{p} / k$.

Within the grating, the field is represented in the Bloch mode expansion

$$
E(x, y)=\sum_{n}\left(a_{n} \cos \left(\beta_{n} z\right)+b_{n} \sin \left(\beta_{n} z\right)\right) u_{n}(x)
$$

where $u_{n}(x)$ is the Bloch mode field satisfying the quasiperiodic Sturm Liouville problem

$$
u_{n}^{\prime \prime}(x)+k^{2} \varepsilon(x) u_{n}(x)=\beta_{n}^{2} u_{n}(x)
$$

where $\varepsilon(x)$ defines the relative permittivity distribution across the lamellar grating profile, i.e., $\varepsilon(x)=\varepsilon_{a}=1$ for $0 \leq x<c_{a}$, and $\varepsilon(x)=\varepsilon_{b}$ for $c_{a} \leq x<c_{a}+c_{b}=d$. While for lossless gratings, the $\left\{u_{n}(x)\right\}$ form a mutually orthogonal set, satisfying the orthogonality relation:

$$
\int_{0}^{d} u_{n}(x) u_{m}(x) d x=\delta_{n m}
$$

the situation for lossy material necessitates the introduction of an adjoint set of modes $\left\{u_{n}^{\dagger}\right\}$ satisfying the same differential equation (20) but with adjoint quasiperiodicity, which are biorthogonal to the primal modes, i.e.,

$$
\int_{0}^{d} u_{n}(x) u_{m}^{\dagger}(x) d x=\delta_{n m}
$$

Accordingly, at the top and bottom interfaces $(z= \pm h / 2)$, the E-field is expressed in the respective forms:

$$
\begin{aligned}
\sum \eta_{p}^{-1 / 2}\left(\delta_{p}+r_{p}\right) e_{p}(x) & =\sum_{n}\left(\tilde{a}_{n}+\tilde{b}_{n}\right) u_{n}(x), \\
\sum \eta_{p}^{-1 / 2} t_{p} e_{p}(x) & =\sum_{n}\left(\tilde{a}_{n}-\tilde{b}_{n}\right) u_{n}(x),
\end{aligned}
$$

respectively. Then, adding and subtracting these to generate the field expression corresponding to symmetric and antisymmetric incidence:

$$
\begin{aligned}
& \sum \eta_{p}^{-1 / 2}\left(s_{p}^{+}+\delta_{p}\right) e_{p}=2 \sum_{n} \tilde{a}_{n} u_{n}(x), \\
& \sum \eta_{p}^{-1 / 2}\left(s_{p}^{-}+\delta_{p}\right) e_{p}=2 \sum_{n} \tilde{b}_{n} u_{n}(x)
\end{aligned}
$$

where $s_{p}^{ \pm}=r_{p} \pm t_{p}$.

To solve the boundary value problem, we need to apply the continuity of the both the tangential components of the electric and magnetic fields at the interface. We therefore need the expression for the field component $H_{x}$ which, for this polarisation, is proportional to $d E / d y$. The corresponding expressions to Eqs (23) and (24) for $d E / d y$ at the top and bottom interfaces are: 


$$
\begin{aligned}
& \sum_{p} i \eta_{p}^{1 / 2}\left(s_{p}^{+}-\delta_{p}\right) e_{p}=2 \sum_{n} D_{1 n} \tilde{a}_{n}^{2} u_{n}(x), \\
& \sum_{p} i \eta_{p}^{1 / 2}\left(s_{p}^{-}-\delta_{p}\right) e_{p}=2 \sum_{n} D_{2 n} \tilde{b}_{n}^{2} u_{n}(x),
\end{aligned}
$$

We then solve the boundary value problems for the symmetric and antisymmetric boundary value problem by a projection method in which we project one of the pair of field matching equations onto the Bloch mode basis of free space, i.e., the plane wave basis $\left\{e_{p}\right\}$, and the other member of the pair onto the Bloch modes of the lamellar structure, i.e., $\left\{u_{n}\right\}$.

For the antisymmetric problem, we project the electric field equation (26) onto the plane wave basis, and the magnetic field equation(28) onto the grating Bloch basis to derive:

$$
\begin{gathered}
s_{-}=-\delta+2 \eta^{1 / 2} J \tilde{b} \\
2 D_{2} \tilde{b}=\mathrm{i} J^{\dagger} \eta^{1 / 2}\left(s_{-}-\delta\right),
\end{gathered}
$$

where $J=\left[J_{p n}\right], J^{\dagger}=\left[J_{n p}^{+}\right]$

$$
\begin{aligned}
& J_{p n}=\int_{0}^{d} e_{p}^{*}(x) u_{n}(x) d x, \\
& J_{n p}^{\dagger}=\int_{0}^{d} e_{p}(x) u_{n}^{\dagger}(x) d x .
\end{aligned}
$$

The completeness of the plane wave and Bloch mode bases, may be expressed in matrix form

$$
\begin{aligned}
& J J^{\dagger}=I, \\
& J^{\dagger} J=I,
\end{aligned}
$$

the derivation of which is straightforward and exploits the orthogonality of the $\left\{e_{p}\right\}$, and the biorthogonality of the $\left\{u_{n}\right\}$ and $\left\{u_{n}^{+}\right\}$.

Returning to the projection equations (29) and (30), we derive

$$
s_{-}+\delta=-B\left(s_{-}-\delta\right)
$$

where $B=-\mathrm{i} \eta^{1 / 2} J^{\mathrm{H}} D_{2}^{-1} J^{\dagger} \eta^{1 / 2}$ and $D_{2}=\operatorname{diag}\left(D_{2 n}\right)$. From this it follows:

$$
s_{-}=(I+B)^{-1}(B-I) \delta .
$$

For the symmetrised problem, it is most convenient to project the field matching equations differently to the manner that was adopted for the antisymmetric problem. This time, we project the magnetic field equation (27) onto the plane wave basis and the electric field equation (25) on the grating Bloch mode basis. This leads to

$$
\begin{aligned}
s^{+}+\delta & =2 \mathrm{i} \eta^{1 / 2} J D_{1} a \\
2 a & =J^{+}\left(\delta+s^{+}\right) .
\end{aligned}
$$

from which it follows

$$
s^{+}-\delta=-A\left(\delta+S^{+}\right),
$$

where $A=\mathrm{i} \eta^{1 / 2} J D_{1} J^{+} \eta^{-1 / 2}$. Accordingly,

$$
\begin{aligned}
s^{+} & =(I+A)^{-1}(I-A) \delta, \\
& =\left[-I+2(I+A)^{-1}\right] \delta .
\end{aligned}
$$

Having now solved the diffraction problem for the lamellar grating in the general case, we now consider the limit of this formulation for ultra-thin, dense gratings. To do so, we consider the limit as the conductivity or the permittivity of the rulings is increased to infinity, i.e., $\varepsilon_{b}=\tilde{\varepsilon} / h \rightarrow \infty$, as the thickness of the grating decreases, i.e., $h \rightarrow 0$. To proceed further, it is necessary to understand the the form of the dominant modes in this limit, i.e., those modes for which the transverse propagation constants $\gamma_{a}$ and $\gamma_{b}$ in each of the two lamellar regions $a$ and $b$ partition into two sets have bounded values, independent of $h$. We begin with the expressions for the transverse propagation constants:

$$
\begin{aligned}
& \gamma_{a}^{2}=k^{2}-\beta^{2}, \\
& \gamma_{b}^{2}=k^{2} \frac{\tilde{\varepsilon}}{h}-\beta^{2},
\end{aligned}
$$

which follow from the Helmholtz equation.

Within the air inclusions, i.e., $\varepsilon_{\mathrm{a}}=1$, the dominant modes have $\gamma_{a}=O\left(h^{0}\right)$, from which it follows that $\beta_{a}=O\left(h^{0}\right)$ and $\gamma_{b}=k^{2} \tilde{\varepsilon} / h=O\left(h^{-1}\right)$. We call these the $a$-type modes and, in fact, can show that $\gamma_{n}^{(a)} \approx n \pi / c_{a}$, and that the modal form is sinusoidal in air and vanishes in the dielectric in the limit as $h \rightarrow 0-$ matching the form of the in-air modes that arise in the formulation for a zero-thickness, perfectly conducting lamellar grating.

Correspondingly, within the dense (lossy) dielectric inclusions, i.e., $\varepsilon_{\mathrm{b}}=\tilde{\varepsilon} / h$, the dominant modes have $\gamma_{b}=O\left(h^{0}\right)$, from which it follows that $\beta_{b}=k \sqrt{\tilde{\varepsilon}} / \sqrt{h}=O\left(h^{-1 / 2}\right)$ and $\gamma_{a}=i k \tilde{\varepsilon} / h=O\left(h^{-1 / 2}\right)$. We call these the $b$-type modes and, in fact, $\gamma_{n}^{(b)} \approx n \pi / c_{b}$, corresponding to a modal form that is sinusoidal in the dielectric and vanishes in air in the limit as $h \rightarrow 0-$ matching the form of the surface currents on the surface of the perfectly conducting strip of zero-thickness.

In this limit, only the $a$ - and $b$-type modes are of interest, in the limit as the grating thickness vanishes and the grating becomes a "grating layer", since all other modes have too high a spatial frequency to couple to the incident field. This partitioning of modes has implications for the completeness relations (33) and (34). Indeed it can be shown that

$$
\begin{aligned}
J_{a} J_{a}^{\dagger}+j_{b} J_{b}^{\dagger} & =I, \\
J_{a}^{\dagger} J_{a} & =I, \\
J_{a}^{\dagger} J_{b} & =0, \\
J_{b}^{\dagger} J_{a} & =0, \\
J_{b}^{\dagger} J_{b} & =I .
\end{aligned}
$$

Further, it can be shown that

$$
J_{b} J_{b}^{\dagger}=F,
$$

where

$$
F_{p q}=\int_{0}^{d} f(x) e_{p}(x) e_{q}^{*}(x) d x
$$

Here, $F$ is a Toeplitz matrix whose elements are derived from the coefficients of a complex Fourier series for

$$
\begin{aligned}
f(x) & =0 & & \text { on interval } a \\
& =1 & & \text { on interval } b,
\end{aligned}
$$

which is useful result that we shall later exploit. 
Having determined the form of the lamellar grating modes, and their partitioning in the grating layer limit, we return now to the understand the asymptotics of the diffraction problem.

For the modes of type $a, \beta_{a}=O\left(h^{0}\right)$, and so

$$
\begin{aligned}
D_{2 a} & =\beta / k_{0} \cot \beta h / 2 \\
& \approx 2 /\left(k_{0} h\right) .
\end{aligned}
$$

Correspondingly, for modes of type $b, \beta_{b}=k_{0} \sqrt{\widetilde{\varepsilon} / h}$, and so

$$
\begin{aligned}
D_{2 b} & =\left(\beta / k_{0}\right) 2 /\left(k_{0} h\right) \\
& \approx 2 /\left(k_{0} h\right) .
\end{aligned}
$$

Accordingly, $D_{2} \rightarrow \infty$ as $h \rightarrow 0$, and so $D_{2}^{-1} \rightarrow 0$, and $B \rightarrow 0$. It therefore follows that for the antisymmetric problem:

$$
s^{-}=-\delta
$$

This result, together with Eq. (41), allows us to infer that

$$
t=\frac{1}{2}\left(s^{+}-s^{-}\right)=T \delta=(I+A)^{-1} \delta .
$$

The asymptotics of the matrix $A$ depends on the matrix $D_{1}=$ $-\left(\beta / k_{0}\right) \tan (\beta h / 2)$. For the modes of type $a, \beta_{a}=O\left(h^{0}\right)$, and so

$$
\begin{aligned}
D_{1 a} & =-\beta / k_{0} \tan \beta h / 2 \\
& \approx-\frac{\beta^{2}}{k} \frac{h}{2} \\
& \rightarrow 0
\end{aligned}
$$

Correspondingly, for modes of type $b, \beta_{b}=k_{0} \sqrt{\tilde{\varepsilon} / h}$, and so

$$
\begin{aligned}
D_{1 b} & =-\beta / k_{0} \tan \beta h / 2 \\
& \approx-\frac{\beta^{2}}{k} \frac{h}{2} \\
& \rightarrow-\frac{k \tilde{\varepsilon}}{2}
\end{aligned}
$$

which is independent of $h$. It therefore follows that

$$
\begin{aligned}
A & =i \eta^{-1 / 2} J D_{1} J^{\dagger} \eta^{-1 / 2} \\
& =i \eta^{-1 / 2}\left(J_{a} D_{1 a} J_{b}^{\dagger}+J_{b} D_{1 b} J_{b}^{\dagger}\right) \eta^{-1 / 2} \\
& \approx-i \frac{k \varepsilon}{2} \eta^{-1 / 2} J_{b} J_{b}^{\dagger} \eta^{-1 / 2} \\
& =\frac{s}{2} \eta^{-1 / 2} F \eta^{-1 / 2}
\end{aligned}
$$

where

$$
\begin{aligned}
s & =-i k \varepsilon \\
\propto & \frac{\lim _{h \rightarrow 0} \epsilon h}{\lambda}
\end{aligned}
$$

In deriving Eq. (66, we have reduced the lamellar grating formulation to the result of grating layer result of Petit and Bouchitte [1], and have elucidated the dependence of the formulation on the factor $\lim _{h \rightarrow 0} \epsilon h / \lambda$ which determines the strength of the grating layer.

While this is the key result, we proceed one step further to derive another form of the transmission scattering matrix (56) with some straightforward manipulation as follows. Proceeding formally:

$$
\begin{aligned}
T= & (I+A)^{-1}=I-A+A^{2}+\ldots \\
= & I-\frac{s}{2} \eta^{-1 / 2} J_{b} J_{b}^{\dagger} \eta^{-1 / 2} \\
& +\frac{s^{2}}{4} \eta^{-1 / 2} J_{b} J_{b}^{\dagger} \eta^{-1 / 2} \eta^{-1 / 2} J_{b} J_{b}^{\dagger} \eta^{-1 / 2}+\ldots \\
= & I-\frac{s}{2} \eta^{-1 / 2} J_{b}\left[I-\frac{s}{2} J_{b}^{\dagger} \eta^{-1} J_{b}+\ldots\right] J_{p}^{\dagger} \eta^{-1 / 2} \\
= & I-\frac{s}{2} \eta^{-1 / 2} J_{b}\left[I+\frac{s}{2} J_{p}^{\dagger} \eta^{-1} J_{b}\right] J_{p}^{\dagger} \eta^{-1 / 2} \\
= & I-\eta^{-1 / 2} J_{b}\left[\frac{2}{s} I+J_{b}^{\dagger} \eta^{-1} J_{b}\right]-1 J_{b}^{\dagger} \eta^{-1 / 2} .
\end{aligned}
$$

In the limit as $s \rightarrow \infty$, we recover the result for the current mode formulation (74) for the strip lamellar grating derived in $[2]$.

$$
T \rightarrow I-\eta^{-1 / 2} J_{b}\left[J_{b}^{\dagger} \eta^{-1} J_{b}\right]^{-1} J_{p}^{\dagger} \eta^{-1 / 2}
$$

Alternatively, if $s=0$, as would occur in the "soft" limit whereby $\lim _{h \rightarrow 0} \varepsilon h=0$, the grating layer would be transparent, consistent with the result from (73) for which $T=I$.

The main result (66) represents the intermediate ground whereby provided that the critical parameter $s$ is bounded, the ultra-thin grating reduces to the grating layer considered by Petit and Bouchitte [1].

\section{REFERENCES}

1. G. Bouchitté and R. Petit, "On the concepts of a perfectly conducting material and of a perfectly conducting and infinitely thin screen," Radio Sci. 24, 13 (1989).

2. L. Botten, R. McPhedran, and G. Milton, "Perfectly Conducting Lamellar Gratings: Babinet's Principle and Circuit Models," J. Mod. Opt. 42, 2453-2473 (1995). 